\title{
Dynamic Contrast Enhanced MRI of the Prostate: Comparison of Gadobutrol and Gd-DTPA
}

\author{
Dynamische kontrastmittelgestützte MRT der Prostata: Vergleich \\ von Gadobutrol mit Gd-DTPA
}

Authors

Affiliations
T. Durmus ${ }^{1}$, B. Vollnberg ${ }^{1}$, C. Schwenke ${ }^{2}$, E. Kilic ${ }^{3}$, A. Huppertz ${ }^{4}$, M. Taupitz ${ }^{1}$, T. Franiel ${ }^{5}$

Department of Radiology, Charité Universitätsmedizin, Berlin, Germany

2 Statistical Consulting, Berlin, Germany, SCO:SSiS, Berlin

3 Department of Pathology, Charité Universitätsmedizin, Berlin, Germany

${ }^{4}$ Charite Berlin, Imaging Science Institute, Berlin

Department of Radiology, Jena University Hospital, Jena, Germany
Key words

- prostate

- MR-imaging

contrast agents

gadolinium

neoplasms

eingereicht 7.1 .2013

akzeptiert $\quad 18.5 .2013$

\section{Bibliography}

DoI http://dx.doi.org/

10.1055/s-0033-1335892

Published online: 25.7.2013

Fortschr Röntgenstr 2013; 185:

862-868 @ Georg Thieme

Verlag KG Stuttgart · New York · ISSN 1438-9029

\section{Correspondence}

Dr. Tahir Durmus

Universitätsmedizin Berlin, Charité Campus Mitte, Institut für Radiologie

Charitéplatz 1

10117 Berlin

Germany

Tel.: +4930450627374

Fax: +4930450527911

Tahir.Durmus@charite.de

\section{Abstract \\ $\nabla$}

Purpose: To evaluate the enhancement profile of the macrocyclic contrast medium (CM) gadobutrol in comparison to linear CM GdDTPA in DCE-MRI of the prostate.

Materials and Methods: In total 53 patients with prostata cancer ( $\mathrm{PCa}$ ) were included, who received a radical prostatectomy after multiparametric MRI of the prostate including DCE-MRI. Using circular regions of interests normal peripheral zone (PZ) and PCa foci $>5 \mathrm{~mm}$ in diameter (42 and 34 foci in Gd-DTPA and gadobutrol group, respectively) were analysed in DCE-MRI. Enhancement curves (Type I, II and III) and pharmacokinetic parameters were analyzed qualitatively and quantitatively and compared using mixed linear models (two sided p-values $<0.05$ were regarded significant).

Results: There was no significant difference in frequencies of curve types I, II or III in the normal PZ ( $p=0.63$ ) or in PCa foci ( $p=0.75)$. PCa with a Gleason score $\geq 7$ had in comparison to Gleason $\leq 6$ significantly more often a Wash-Out-curve (Type III) with both CM $(p=0.02)$. The relative peak enhancement was in the PZ (Gd-DTPA 1.4 a. u. [1.20; 1.59], gadobutrol 1.58 a. u. $[1.37 ; 1.78])$ and in PCa foci (Gd-DTPA 1.56 a. u. [1.41; 1.71$]$, gadobutrol 1.76 a. u. [1.59; 1.94$]$ ) significantly higher with gadobutrol $(p=0.04)$. The pharmacokinetic parameters $K^{\text {trans }}$ und $k_{e p}$ were higher in PCa foci than in PZ $(\mathrm{p}<0.0001$ and $\mathrm{p}=0.002$, respectively) without significant difference of the parameter values between both $\mathrm{CM}$ $(\mathrm{p}=0.65)$.

Conlusion: This study is the first systematic comparison of gadobutrol and Gd-DTPA in DCE-MRI of the prostate. The relative peak enhancement is higher using gadobutrol com- pared to Gd-DTPA in DCE-MRI. There was no statistically significant difference in curve types or the pharmacokinetic parameters in PCa or normal PZ between both CM.

Key Points:

- Gadobutrol yields a higher and faster peak enhancement in prostate cancer and in the normal peripheral zone compared to GdDTPA.

- There was no statistically significant difference observed in curve type frequencies and pharmacokinetic parameters between both CM.

- Gadobutrol as Gd-DTPA appear to be suitable for DCE-MRI of the prostate for prostate cancer detection.

Citation Format:

- Durmus T, Vollnberg B, Schwenke C etal. Dynamic Contrast Enhanced MRI of the Prostate: Comparison of Gadobutrol and Gd-DTPA. Fortschr Röntgenstr 2013; $862-868$

\section{Zusammenfassung}

$\nabla$

Ziel: Vergleich des makrozyklischen Kontrastmittels (KM) Gadobutrol und des linearen KM Gd-DTPA bezüglich des Signalintensitätsverlaufs in der dynamischen Kontrastmitteluntersuchung (DCE-MRI) der Prostata.

Material und Methoden: Es wurden insgesamt 53 Patienten mit Prostatakarzinom (PCa) eingeschlossen, welche nach einer multiparametrischen MRT der Prostata mit DCE-MRI prostatektomiert wurden. Jedes PCa $>5 \mathrm{~mm}$ (42 PCa in GdDTPA-Gruppe, 34 PCa in Gadobutrol-Gruppe) und die gesunde periphere Zone (PZ) wurden in der DCE-MRI qualitativ und quantitativ analysiert. Kurvenverläufe (TypI, II und III) und pharmakokinetische Parameter $K^{\text {trans }}$ und $k_{\text {ep }}$ wurden anhand 
gemischt linearer Modelle zwischen beiden Gruppen verglichen; zweiseitige p-Werte $<0,05$ wurden als signifikant definiert.

Ergebnisse: Die Frequenz der Kurventypen I, II und III unterschied sich zwischen den KM weder in der PZ $(p=0,63)$ noch im PCa $(p=0,75)$ signifikant voneinander. PCa mit einem GleasonScore $\geq 7$ zeigten im Vergleich zu Gleason-Score $\leq 6$ statistisch signifikant häufiger bei beiden KM eine Wash-out-Kurve (Typ III, $\mathrm{p}=0,02)$. Das relative Signalintensitätsmaximum war in der PZ (Gd-DTPA 1,4 a.u. [1,20; 1,59], Gadobutrol 1,58 a. u. [1,37; $1,78]$ ) und im PCa-Gewebe (Gd-DTPA 1,56 a. u. [1,41; 1,71], Gadobutrol 1,76 a. u. $[1,59 ; 1,94])$ für Gadobutrol signifikant höher $(\mathrm{p}=0,04)$. Die pharmakokinetischen Parameter $\mathrm{K}^{\text {trans }}$ und $\mathrm{k}_{\mathrm{ep}}$ waren im Tumorgewebe signifikant höher als in der PZ ( $\left.\mathrm{K}^{\text {transp }}<0,0001 ; \mathrm{k}_{\text {ep }} \mathrm{p}=0,002\right)$. Zwischen den $\mathrm{KM}$ fand sich kein signifikanter Unterschied bezüglich der pharmakokinetischen Parameter $(\mathrm{p}=0,65)$

Schlussfolgerung: Diese Arbeit ist der erste systematische Vergleich von Gadobutrol und Gd-DTPA in der DCE-MRI der Prostata. Hierbei konnte gezeigt werden, dass das maximale relative Enhancement mit Gadobutrol im Vergleich zu Gd-DTPA höher ist, wobei Kurventypen und pharmakokinetische Parameter $\mathrm{K}^{\text {trans }}$ und $\mathrm{k}_{\mathrm{ep}}$ weder im PCa noch in der normalen PZ einen statistisch signifikanten Unterschied aufweisen.

\section{Introduction}

$\nabla$

Cancer of the prostate is the most frequent malignant tumor in men, and, after cancer of the lung and colon, has the third-highest mortality rate. The diagnosis of prostate cancer is based on digital-rectal examination, determination of the prostate-specific antigen (PSA) and transrectal ultrasound-guided (TRUS) biopsy. In the case of TRUS-guided biopsy, a total of 10 - 12 samples are taken from both lateral lobes of the prostate following a systematic pattern. Nevertheless, in the case of $20-40 \%$ of patients suspected of prostate cancer, a definitive diagnosis of cancer can be ascertained only after repeated biopsy, since a TRUS biopsy is limited by sampling error $[1,2]$. This represents a diagnostic problem, since a raised or increasing PSA value raises the suspicion of prostate cancer; however the low negative predictive value of a TRUS biopsy cannot definitively indicate that the patient actually has no cancer. A multiparametric MRI has been shown to be a very sensitive and specific non-invasive method of localizing potentially cancerous lesions within the prostate [3, 4]. Multiparametric MRI includes conventional T2- and T1-weighted imagining combined with diffusion imaging and or ${ }^{1} \mathrm{H}$-magnetic resonance spectroscopy and/or dynamic contrast-enhanced MR imaging (DCE-MRI). Using this approach, T2-weighted imaging is indispensable, especially for interpretation of changes in the transition zone [5]. Compared to T2-weighted imaging alone DCE-MRI provides a diagnostic advantage with respect to prostate cancer detection [6, 7]. Essential in this regard is dynamic information pertaining to contrast medium inflow, since generally T1-weighted gradient echo sequences are used with a brief resolution of $<5 \mathrm{~s} /$ measurement [8].

The first approved gadolinium-containing contrast medium for MR diagnosis was gadopentetate dimeglumine (GdDTPA; Magnevist, Bayer Healthcare, Berlin, Germany). Consequently, Gd-DTPA was the contrast medium of choice for
DCE-MRI of the prostate, as it was for all other organ systems $[3,7,9]$. Since the introduction of Gd-DTPA, a number of additional contrast agents have been approved which are distinguished by their chemical properties. Consequently, studies have been required to test these contrast media and compare them with respect to their relative advantages and disadvantages [10-13]. For safety reasons, the occurrence of nephrogenic systemic fibrosis (NSF) accompanying the use of MR contrast media with linear complexes brought to the forefront more thermodynamically stable contrast media with macrocyclic complexes [14, 15]. Todate, there has been no systematic comparison of the use of macrocyclic and linear contrast media in prostate DCEMRI, and therefore no data indicating whether the application of gadobutrol is as reliable as Gd-DTPA in the diagnosis of prostate cancer. Gadobutrol differs from Gd-DTPA by its macrocyclic structure, charge neutrality, its doubled molarity as well as a higher T1 relativity (Gd-DTPA r1 $=4.1 \mathrm{mmol}^{-}$ ${ }^{1} \mathrm{~s}^{-1}$, Gadobutrol $\left.\mathrm{r} 1=5.2 \mathrm{mmol}^{-1} \mathrm{~s}^{-1}\right)[10,16]$. T1 relativity of a substance is a measure of the signal-increasing effect on T1weighted sequences, as already demonstrated in numerous studies of various organ systems [17-20]. The purpose of this study is to delineate the differences between both contrast media in their enhancement behavior according to contrast medium dosage and the diagnostic value of the prostate DCE MRI based on this comparison, as well as the evaluation of the signal intensity time (SI-t) progression of the macrocyclic contrast medium gadobutrol with the most commonly agent Gd-DTPA in prostate DCE-MRI. Proceeding from results of studies of other organ systems [12, 19, 20], this study is based on the hypothesis that gadobutrol provides a higher relative enhancement in prostate tissue, but that there is no qualitative difference in the enhancement progression.

\section{Materials and Methods \\ $\nabla$}

\section{Study Population}

These studies included patients with histologically verified cancer of the prostate who were examined between 1/2009 and 12/2010 with multiparametric MRI of the prostate using Gd-DTPA or gadobutrol with 1.5 T (Magnetom Avanto, Siemens, Erlangen, Germany), and who subsequently underwent prostatectomy. Inclusion criteria in this retrospective study were prostatectomy after histologic verification of prostate cancer, a multiparametric MRI performed according to standardized institute protocol; examination was performed within a week prior to the prostatectomy; dosage of Gd-DTPA at a flow rate of $4 \mathrm{ml} / \mathrm{s}$ or gadobutrol at $2 \mathrm{ml} / \mathrm{s}$ for the DCE-MRI. An additional inclusion criterion was a carcinoma map created by a consensus of the pathologist and radiologist based upon the pathological prostate sample. After the prostatectomy the entire prostate sample was marked in color (anterior, posterior, left, right) and sliced perpendicular to the longitudinal axis from the apex to the base ( $5-8$ slices). The individual slices were quartered into individual slices according to standard protocols, embedded in paraffin and stained with hematoxylin and eosin. All pathologically verified carcinomas $>5 \mathrm{~mm}$ as well as areas with a normal peripheral zone were graphically transferred to standardized drawings by the pathologist. 
All tumor patterns were assessed using the modified Gleason Scoring System [21]. Then, based upon the consensus of the pathologist and radiologist, the carcinoma foci and normal peripheral zone were separately plotted and coded on printed $\mathrm{T} 2 \mathrm{w}$ images. As needed, anatomical landmarks were relied upon for exact identification of the tumor foci on the most closely corresponding MR images; these included zonal structures, prostatic urethra, bladder, ejaculatory duct and seminal vesicles. The serum creatinine and serum PSA values were determined in all patients prior to the MRI examination. The local ethics commission approved this study. Altogether 28 patients were included in the study who had been examined using Gd-DTPA (Magnevist; Bayer Healthcare, Berlin, Germany) - hereafter designated Group A, and 25 patients who had been examined using gadobutrol (Gadovist; Bayer Healthcare, Berlin, Germany) - hereafter designated Group B. A normally classified T2w hyperintense area in the peripheral zone of all patients was previously analyzed in the Pathology department. In addition, 42 cancerous areas in Group A and 34 in Group B were assessed. The median age in Group A (Gd-DTPA) was 62.0 5 5.9 years, and $62.3 \pm 5.7$ years in Group B (gadobutrol). The PSA value in both groups was not statistically different $(p>0.05)$, with an average value of $8.6 \pm 4.8 \mathrm{ng} / \mathrm{ml}$ for Group A, and $9.7 \pm 7.6 \mathrm{ng} /$ $\mathrm{ml}$ for Group B. The median Gleason score for prostate cancer was 7 in both groups.

\section{Multiparametric MRI with Dynamic Contrast Medium Examination}

Using a combined 6-channel surface coil and an endorectal coil (Medrad Prostate eCoil ${ }^{\mathrm{TM}}$, Bayer Healthcare, Berlin, Germany), all patients received a multiparametric MRI of the prostate according to an identical examination protocol by means of a commercially-available MRI unit (1.5 T Magnetom Avanto, Siemens, Erlangen, Germany). After localizer sequences, the prostate was examined using T2-weighted Turbo-Spin Echo (TSE) sequences in axial orientation (repetition time/echo time in msec, 4850/85; echo train length, 15; number of signals acquired, 3; field of view, $160 \times 160 \mathrm{~mm}$ ) and coronal scan orientation. In addition, a T1-weighted sequence was measured across the prostate in the axial direction (691/12; echo train length, 3; number of signals acquired, 2; field of view, $160 \times 160 \mathrm{~mm}$ ). All sequences were measured using a matrix of $256 \times 256$, section thickness of
$3.0 \mathrm{~mm}$, slice increment of $0.6 \mathrm{~mm}$, and $100 \%$ phase oversampling.

Maintaining the angulation of the T2-TSE, a diffusionweighted as well as a DCE-MRI were additionally performed. In order to calculate longitudinal relaxation rate R10 [22, 23], a gradient echo sequence was measured with the following parameters, each with a flip angle of $2^{\circ}, 5^{\circ}, 10^{\circ}$, and $15^{\circ}$ (4.57/1.63; number of signals acquired, 5; field of view, $260 \times 260 \mathrm{~mm}$; image matrix, $256 \times 256$; section thickness, $3.6 \mathrm{~mm}$ ). The DCE-MRI was performed using a fast gradient echo sequence was measured with a temporal resolution of 3.9 seconds (5.19/2.02; flip angle, $15^{\circ}$; number of signals acquired, 1; field of view, $260 \times 260 \mathrm{~mm}$; image matrix, $256 \times 256$; section thickness, $3.6 \mathrm{~mm}$ ). In total, 75 individual measurements were acquired; after the third measurement, the contrast medium was adapted to body weight $(0.1 \mathrm{mmol} /$ $\mathrm{kg}$ body weight) and applied intravenously as a bolus. Since gadobutrol $1 \mathrm{M}$ is double-concentrated compared to GdDTPA, a patient weighing $70 \mathrm{~kg}$ received $7 \mathrm{ml}$ gadobutrol and $14 \mathrm{ml} \mathrm{Gd-DTPA}$ applied intravenously. In order to reconcile the injection time of both groups, gadobutrol was injected using a halved flow of $2.0 \mathrm{ml} / \mathrm{s}$ compared to a rate of $4 \mathrm{ml} /$ $\mathrm{s}$ using Gd-DTPA. After injection of the contrast medium, there was an injection of $20 \mathrm{ml}$ isotonic saline solution while maintaining the flow rates.

Using the open two-compartment model according to Tofts and Kermode [24], the pharmacokinetic parameter maps were calculated for the exchange components $\mathrm{K}^{\text {trans }}$ and $\mathrm{k}_{\mathrm{ep}}$.

\section{Evaluation}

Using evaluation software (syngo Tissue 4 D, Siemens, Erlangen, Germany) the DCE-MRI was registered to the morphological images (T2w) in order to exactly localize the tumor area and normal peripheral zone based on the carcinoma map.Circular fields were used to indicate tumor patterns and normal peripheral zones (BV). The SI-t enhancement curves in the marked fields were calculated as a relative SI- $t$ enhancement of the initial SI without contrast medium, and the individual values were exported over time to an external computer. In addition, the 3-dimensional pharmacokinetic parameter maps of the prostate were calculated using the volume element marking containing the entire prostate. Parameters $\mathrm{K}^{\text {trans }}$ and $\mathrm{k}_{\text {ep }}$ were determined in the previous established fields and likewise exported ( $\bullet$ Fig. 1 ).
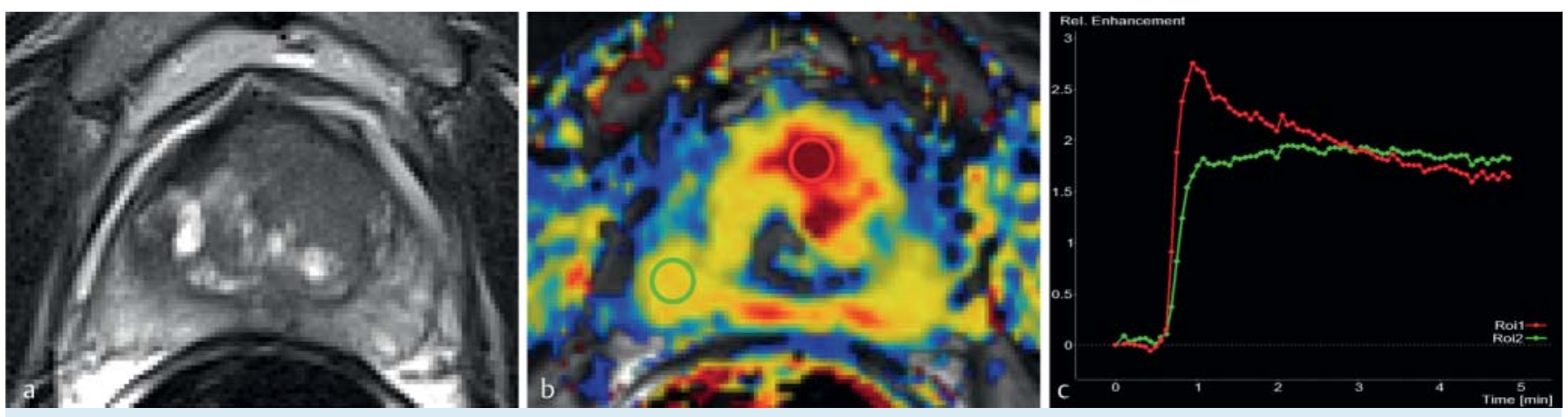

measure relative enhancement in DCE-MRI and calculate pharmacokinetic parameter maps - Ktrans-parameter map shown here. c The resulting enhancement-curve in the peripheral zone (type II, plateau) and the PCa (type III, wash-out). 
Based on the values for the relative SI enhancement of the tumors and healthy peripheral zone per time unit, all curves per patient were calculated, blinded and printed out. A radiologist (TD) categorized all curves as Type I, II or III, whereby a Type I curve reflected a steady increase of the enhancement progression; Type II reflected a plateau, and Type III corresponded to a decline in relative enhancement after the peak [25]. These results were then designated (tumor, normal tissue in the peripheral zone, contrast medium) and statistically evaluated [CS, TD].

\section{Statistics}

Quantitative data are provided as the average of the least squares from mixed linear models with $95 \%$ confidence intervals. Qualitative data are represented as absolute and relative frequencies. The curves between the contrast media were analyzed using multinomial regression analysis in order to compensate for repeated observations of a patient. Relative peak enhancement was defined as the maximum enhancement in the temporal progression of dynamic measurement. Using mixed linear models, this was compared with variance components. Tissue types (tumor, normal peripheral zone), contrast medium and the interaction of both factors served as cofactors. Using the Wilcoxon ranksum test, the two contrast agents were compared with one another. The temporal curve progressions were compared using linear models including all individual measured values over the entire duration of the measurement. Analogously, the pharmacokinetic parameters $\mathrm{K}^{\text {trans }}$ and $\mathrm{k}_{\mathrm{ep}}$ were compared between the contrast media. Here again, tissue type and interaction of tissue type and contrast medium served as cofactors. In a further analysis, the tumors were examined under stratification in Gleason score $\leq 6$ and $\geq 7$. All tests were performed bilaterally, p-values $<0.05$ were considered significant. SAS 9.2 (SAS Institute Inc., Cary, NC, USA) was used to perform the analyses.

\section{Results}

$\nabla$

Qualitative Analysis: Increase (Type I), Plateau (Type II) and Wash-out (Type III)

Statistically, the frequency of curve types between the contrast media did not differ ( $p=0.63$; 0 Table 1$)$. In the normal peripheral zone a Type I curve (steady increase) was shown in $46.4 \%$ of cases in Group A, and in $48.0 \%$ of cases in Group B. A Type II curve (Plateau) was ascertained among $53.6 \%$ (Group A) and 52.0\% (Group B). No Type III curve (Washout) could be observed in either Group A (Gd-DTPA) or Group B (gadobutrol). In $20.6 \%$ of the cases, gadobutrol resulted in a wash-out in the tumor, whereas with Gd-DTPA, a $16.3 \%$ rate was not significantly lower. Tumors in Group B (gadobutrol) more frequently demonstrated a Type II curve than in Group A (Gd-DTPA) and correspondingly fewer Type I curves; this was, however, statistically insignificant ( $\mathrm{p}=0.75$; 0 Table 1$)$.

In addition, the tumors, based on Gleason scores, were divided into two groups (Gleason score $\leq 6$ and $\geq 7$ ) in order to evaluate possible differences between the contrast media in relation to Gleason scores. Consequently it was shown that use of both contrast agents achieved a very similar frequency distribution with respect to curve types; there were no significant differences between the contrast media for tumors with a Gleason score of $\leq 6$ or $\geq 7$ ( Table 2, $\mathrm{p}=0.43$ ). Tumors with a Gleason score of $\geq 7$, compared to tumors with a score of $\leq 6$, more frequently demonstrated a statistically significant Type III curve for both contrast media ( $\triangle$ Table 2, $\mathrm{p}=0.02$ ).

\section{Quantitative Analysis: Relative Peak Enhancement and Curve Gradient}

The relative SI maximum in normal tissue was 1.4 times $[1.20 ; 1.59]$ the initial value in the Gd-DTPA group, whereas this value in the gadobutrol group was significantly higher, with 1.58 [1.37; 1.78$](\mathrm{p}=0.04)$. This behavior was similar in tumor tissue. When Gd-DTPA was used, an average peak value of 1.56 [ $1.41 ; 1.71]$ was achieved, whereas with gadobutrol a statistically significant higher value of 1.76 [1.59; 1.94] was achieved $(p=0.04$, $\diamond$ Table 3$)$. These differences were additionally analyzed using a mixed linear model with the inclusion of all measured values in the entire temporal progression. In this case, no distinction could be established between the contrast media in the normal peripheral zone. However, in tumor tissue a trend toward higher relative enhancement values was evident across the entire temporal progression when gadobutrol $(\mathrm{p}=0.05)$ was used ( $\bullet$ Fig. 2). For both tissue types, the shapes of the curves (interaction of contrast agent and time) for GdDTPA and gadobutrol varied significantly (peripheral zone $\mathrm{p}=0.003$, tumor tissue $\mathrm{p}=0.0003$ ). Observation of the resulting curves in the different tissues makes it clear that

\begin{tabular}{|c|c|c|c|c|c|}
\hline & & type I & type II & type III & p-value \\
\hline Gd-DTPA & \multirow[t]{2}{*}{$\mathrm{PZ}$} & $13(46.4)$ & $15(53.6)$ & 0 & \multirow[t]{4}{*}{0.75} \\
\hline Gadobutrol & & $12(48.0)$ & $13(52.0)$ & 0 & \\
\hline Gd-DTPA & \multirow[t]{3}{*}{ PCa } & $21(48.8)$ & $15(34.8)$ & $7(16.3)$ & \\
\hline Gadobutrol & & $12(35.3)$ & $15(44.1)$ & $7(20.6)$ & \\
\hline p-value & & 0.63 & & & \\
\hline
\end{tabular}

Distribution of enhancement curves of Type I, II and III in normal peripheral zone (PZ) and prostate cancer (PCa) using Gd-DTPA and gadobutrol; absolute number and percent in parentheses.

\begin{tabular}{|c|c|c|c|c|c|c|c|}
\hline & & type I & type II & type III & p-value KM & p-value Gleason & Table 2 Curve types according \\
\hline Gd-DTPA & \multirow[t]{2}{*}{$\mathrm{GS} \leq 6$} & $24(53.3)$ & $19(42.2)$ & $2(4.4)$ & \multirow[t]{4}{*}{0.43} & \multirow[t]{4}{*}{0.02} & to Gleasc \\
\hline Gadobutrol & & $18(45.0)$ & $19(47.5)$ & $3(7.5)$ & & & \\
\hline Gd-DTPA & \multirow[t]{2}{*}{$\mathrm{GS} \leq 7$} & $10(38.5)$ & $11(42.3)$ & $5(19.2)$ & & & \\
\hline Gadobutrol & & $6(31.6)$ & $9(47.4)$ & $4(21.5)$ & & & \\
\hline
\end{tabular}

Frequency of enhancement curve Types I, II and III in prostate cancer of Gleason score (GS) $\leq 6$ and $\geq 7$ using Gd-DTPA and gadobutrol, absolute number and percent in parentheses. 
the rise of the enhancement curve when gadobutrol is used is greater than when Gd-DTPA is utilized, although the further progression is the same (except for a parallel shift upward in the case of the gadobutrol tumor enhancement curve), as shown in $\bullet$ Fig. 2.

Pharmacokinetic Parameters $\mathbf{K}^{\text {trans }}$ and $\mathbf{k}_{\mathrm{ep}}$

For all patients the pharmacokinetic parameters $\mathrm{K}^{\text {trans }}$ and $\mathrm{k}_{\mathrm{ep}}$ were compared between the contrast media. No statistically significant differences for $\mathrm{K}^{\text {trans }}$ and $\mathrm{k}_{\mathrm{ep}}$ could be determined for the contrast agents $\left(K^{\text {trans }} \mathrm{p}\right.$-value $=0.41, \mathrm{k}_{\mathrm{ep}}$ p-value $=0.65)(\diamond$ Table 4$)$. The use of Gd-DTPA as well as gadobutrol demonstrated significantly higher pharmacokinetic parameters $\mathrm{K}^{\text {trans }}$ and $\mathrm{k}_{\mathrm{ep}}$ in tumor tissue than in normal tissue ( $\triangle$ Table $4, K^{\text {trans }} \mathrm{p}$-value $<0.0001 ; k_{\text {ep }}$ p-value

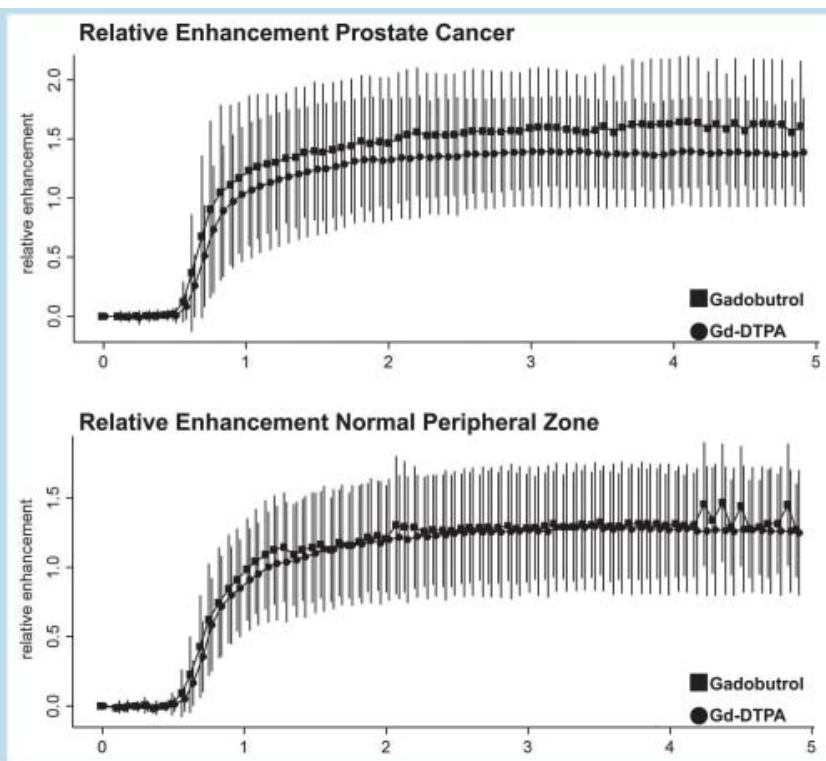

Fig. 2 Relative enhancement in prostate cancer (PCA) and normal peripheral zone tissue using gadobutrol and Gd-DTPA. Peak enhancement was significantly higher using gadobutrol in both PCa and the normal peripheral zone $(p=0.04)$. Comparing the entire observation period, a trend towards higher enhancement using gadobutrol was observed in PCa but not in the peripheral zone. The curve shapes were significantly different caused by a faster enhancement increase using gadobutrol (peripheral zone $p=0.003$; PCa $\mathrm{p}=0.0003)$.

Table 3 Peak enhancement.

\begin{tabular}{|llll|}
\hline & PZ & PCa & P-value \\
\cline { 1 - 3 } Gd-DTPA & $1.40[1.20 ; 1.59]$ & $1.56[1.41 ; 1.71]$ & 0.04 \\
Gadobutrol & $1.58[1.37 ; 1.78]$ & $1.76[1.59 ; 1.94]$ & \\
\hline
\end{tabular}

Relative peak enhancement in DCE-MRI using Gd-DTPA and gadobutrol in normal peripheral zone (PZ) and prostate cancer (PCa).
0.002). Further, when considering the Gleason categories, parameter $\mathrm{K}^{\text {trans }}$ for tumors with a Gleason score of $\geq 7$ when Gd-DTPA is used (0.12 [0.09; 0.15]) and gadobutrol $(0.14$ [0.10;0.17]) was significantly higher than for tumors with a Gleason score of $\leq 6$ (Gd-DTPA $(0.082$ [0.04; 0.12] and gadobutrol (0.079 [0.04; 0.12]); $\mathrm{p}=0.02)$; however, once again there was no statistically significant difference between the contrast media $(\mathrm{p}=0.60)$.

\section{Discussion}

A comparison of various MR contrast media with respect to SI-t progression and detection of lesions had previously been performed in neuroradiological and cardiovascular magnetic resonance imaging as well as in MR mammography $[12,18-20]$. A potential difference of MR contrast agents in DCE-MRI of the prostate had not been previously performed. Therefore, the purpose of this investigation was to analyze the use of gadobutrol and Gd-DTPA in DCE-MRI in order to evaluate possible effects on the resulting curve types and pharmacokinetic parameters.

Several characteristics principally differentiate gadobutrol and Gd-DTPA. While Gd-DTPA is a linear and ionic magnetic resonance contrast medium, gadobutrol has a macrocyclic structure and is charge-neutral [10]. Gd-DTPA has a concentration of $0.5 \mathrm{M}$, whereas gadobutrol has a $1 \mathrm{M}$ concentration. Thus, examinations using gadobutrol were performed using half the flow compared to DCE-MRI with Gd-DTPA in order to administer the identical volume of contrast medium during the same time period. In addition, gadobutrol has about one-fourth higher $\mathrm{T} 1$ relaxivity $(1.5 \mathrm{~T})$ compared to Gd-DTPA [16].

Compared to examinations using Gd-DTPA, the current study determined that the gadobutrol-enhanced DCE-MRI demonstrated a higher relative peak enhancement in the healthy peripheral zone of the prostate and tumor tissues. Taking into account all measured values over the entire time progression, a greater increase in the relative enhancement curve was observed in the gadobutrol group, in addition to a trend toward a higher relative enhancement. These differences can be explained by the higher T1 relaxivity of gadobutrol. This had already been described in magnetic resonance mammography, MRI of brain metastases or infectious lesions, as well as in MR imaging using gadobutrol and other extracellular contrast media with higher relaxivity $[12,19,20]$. Additional factors such as charge differences of each contrast medium are also considered as potential influential factors for enhancement behavior. However, charge differences are primarily relevant in so-called delayed imaging, e.g. in cartilage imaging (dGEMRIC) [26]. Within this study we observed SI-t progression of the DCEMRI for about 5 minutes immediately after administration

\begin{tabular}{|c|c|c|c|c|c|}
\hline & & PZ & PCa & p-value KM & p-value tissue \\
\hline Gd-DTPA & \multirow[t]{2}{*}{$\mathrm{K}^{\text {trans }}$} & $0.05[0.03 ; 0.07]$ & $0.11[0.09 ; 0.12]$ & \multirow[t]{2}{*}{0.408} & \multirow[t]{2}{*}{$<0.0001$} \\
\hline Gadobutrol & & $0.06[0.04 ; 0.09]$ & $0.11[0,09 ; 0,13]$ & & \\
\hline Gd-DTPA & \multirow[t]{2}{*}{$k_{\text {ep }}$} & $0.20[0.13 ; 0.27]$ & $0.31[0.25 ; 0.37]$ & \multirow[t]{2}{*}{0.651} & \multirow[t]{2}{*}{0.002} \\
\hline Gadobutrol & & $0.22[0.14 ; 0.30]$ & $0.33[0.26 ; 0.39]$ & & \\
\hline
\end{tabular}

Table 4 Pharmacokinetic parameters.

Pharmacokinetic parameters ( $\mathrm{K}^{\text {trans }}$ und $\mathrm{k}_{\mathrm{ep}}$ ) in normal peripheral zone (PZ) and prostate cancer (PCa) using Gd-DTPA and gadobutrol. 
of the contrast medium. We primarily observed differences in peak enhancement values and increase of the initial enhancement, so that an effect of the charge differences between both contrast agents is probably negligible in this context.

Differences were also found regarding the number of detected lesions during imaging of cerebral lesions of mammary carcinomas $[20,27,28]$. This was not reviewed in the current study. In our opinion, with respect to multiparametric MRI of the prostate, DCE-MRI primarily aids in increasing the specificity as well as the detection and characterization of the most aggressive lesion [29-31]. In this investigation we could not establish a difference in frequency among curve types I, II or III. Based on our data, we presume that both contrast media do not qualitatively differ from one another to a clinically relevant extent in either a healthy prostate or tumor tissue with respect to enhancement behavior, since the resulting curve types in both groups exhibit similar frequency. Also in comparing Gleason scores, no statistically significant differences could be determined between the two groups. These results are substantiated by a comparative analysis of the pharmacokinetic parameters. With respect to $\mathrm{K}^{\text {trans }}$ and $\mathrm{k}_{\mathrm{ep}}$ values, no statistical difference between these contrast agents could be found.

The study additionally showed that $48.8 \%$ of tumors in Group A (Gd-DTPA) and 35.3\% in Group B (gadobutrol) demonstrated a Type I curve. Of these $38.5 \%$ of tumors in Group A and 31.6\% in Group B had a Gleason score of $\geq 7$. In the recently published ESUR guidelines for structured assessment and classification of prostate carcinomas, suspected tumorous areas are graded from 1 (significant carcinoma highly unlikely) to 5 (clinically significant carcinoma highly likely) [32, 33]. The purpose of this study was not to evaluate the PIRADS criteria. Nevertheless, it should be pointed out that more than $30 \%$ of tumors with a Gleason score of $\geq 7$ demonstrate a Type I curve, which, according to the current PIRADS classification in DCE-MRI is only assigned a point value of 1 . Additional criteria such as focality and asymmetry are only provided for a Type II or Type III curve [32]. During a pending evaluation of PIRADS criteria, therefore, these additional criteria should also be applied to tumors showing a Type I curve, analogously to magnetic resonance mammography [34].

This study is limited by its retrospective approach and interindividual comparison. However, all patients undergoing prostatectomy are treated so that for each carcinoma the best available histological standard is applied with respect to diagnosis and Gleason grading. Due to the required double contrast medium dose and the resulting time delay, intraindividual comparison is difficult to perform in the case of patients with histologically verified carcinoma and scheduled prostatectomy.

On the whole it can be concluded that both gadobutrol and Gd-DTPA appear to be highly suitable for DCE-MRI. Relative enhancement in DCE-MRI using gadobutrol tends to be higher; in the course of this study no statistically significant difference could be found with respect to curve type frequencies and the pharmacokinetic parameters $\mathrm{K}^{\text {trans }}$ and $\mathrm{k}_{\mathrm{ep}}$.

\section{Clinical Relevance}

\section{$\nabla$}

No statistically significant differences were found between both contrast medium types in the curve type frequencies and pharmacokinetic parameters.

Both the macrocyclic gadobutrol and the linear contrast agent Gd-DTPA appear to appear to be well-suited for DCEMRI of the prostate for the diagnosis of prostate cancer.

\section{Acknowledgement}

\section{$\nabla$}

This manuscript is dedicated to Professor Bernd Hamm for his 60th birthday.

\section{Literatur}

1 Hong YM, Lai FC, Chon CH et al. Impact of prior biopsy scheme on pathologic features of cancers detected on repeat biopsies. Urol Oncol 2004; 22: 7-10

2 Djavan B, Ravery V, Zlotta A et al. Prospective evaluation of prostate cancer detected on biopsies 1, 2, 3 and 4: when should we stop? J Urol 2001; 166: 1679-1683

3 Turkbey B, Mani H, Shah V et al. Multiparametric 3T prostate magnetic resonance imaging to detect cancer: histopathological correlation using prostatectomy specimens processed in customized magnetic resonance imaging based molds. J Urol 2011; 186: 1818-1824

4 Roethke M, Anastasiadis AG, Lichy M et al. MRI-guided prostate biopsy detects clinically significant cancer: analysis of a cohort of 100 patients after previous negative TRUS biopsy. World J Urol 2012; 2: 213-218

5 Hoeks CM, Hambrock T, Yakar D et al. Transition zone prostate cancer: detection and localization with 3-T multiparametric MR imaging. Radiology 2013; 266: 207-217

6 Futterer JJ, Heijmink SW, Scheenen TW et al. Prostate cancer localization with dynamic contrast-enhanced MR imaging and proton MR spectroscopic imaging. Radiology 2006; 241: 449-458

7 Franiel T, Stephan C, Erbersdobler A et al. Areas suspicious for prostate cancer: MR-guided biopsy in patients with at least one transrectal USguided biopsy with a negative finding - multiparametric MR imaging for detection and biopsy planning. Radiology 2011; 259: $162-172$

8 Franiel T, Hamm B, Hricak H. Dynamic contrast-enhanced magnetic resonance imaging and pharmacokinetic models in prostate cancer. Eur Radiol 2011; 21: 616-626

9 Hoeks CM, Barentsz JO, Hambrock T et al. Prostate cancer: multiparametric MR imaging for detection, localization, and staging. Radiology 2011; 261: 46-66

10 Tombach B, Heindel $W$. Value of 1.0-M gadolinium chelates: review of preclinical and clinical data on gadobutrol. Eur Radiol 2002; 12: $1550-1556$

11 Allard M, Doucet D, Kien P et al. Experimental study of DOTA-gadolinium. Pharmacokinetics and pharmacologic properties. Invest Radiol 1988; 23: S271-S274

12 Attenberger UI, Runge VM, Morelli JN et al. Evaluation of gadobutrol, a macrocyclic, nonionic gadolinium chelate in a brain glioma model: comparison with gadoterate meglumine and gadopentetate dimeglumine at $1.5 \mathrm{~T}$, combined with an assessment of field strength dependence, specifically 1.5 versus 3 T. J Magn Reson Imaging 2010; 31 : 549-555

13 Chanalet S, Masson B, Boyer L et al. Etudes comparatives de la tolerance du gadodiamide, du gadopentetate de dimeglumine et du gadoterate de meglumine au cours d'un examen IRM du systeme nerveux central. J Radiol 1995; 76: 417 - 421

14 Frenzel T, Lengsfeld $P$, Schirmer $H$ et al. Stability of gadolinium-based magnetic resonance imaging contrast agents in human serum at 37 degrees C. Invest Radiol 2008; 43: 817-828

15 Abujudeh $H H$, Kaewlai R, Kagan A et al. Nephrogenic systemic fibrosis after gadopentetate dimeglumine exposure: case series of 36 patients. Radiology 2009; 253: 81 - 89

16 Rohrer M, Bauer H, Mintorovitch J et al. Comparison of magnetic properties of MRI contrast media solutions at different magnetic field strengths. Invest Radiol 2005; 40: 715-724 
17 Tweedle MF, Wedeking P, Telser J et al. Dependence of MR signal intensity on Gd tissue concentration over a broad dose range. Magn Reson Med 1991; 22: 191 - 194 discussion 195 - 196

18 Herborn CU, Lauenstein TC, Ruehm SG et al. Intraindividual comparison of gadopentetate dimeglumine, gadobenate dimeglumine, and gadobutrol for pelvic 3D magnetic resonance angiography. Invest Radiol 2003; 38: $27-33$

19 Durmus T, Schilling R, Doeblin P et al. Gadobutrol for magnetic resonance imaging of chronic myocardial infarction: intraindividual comparison with gadopentetate dimeglumine. Invest Radiol 2012; 47: $183-188$

20 Martincich L, Faivre-Pierret M, Zechmann CM et al. Multicenter, doubleblind, randomized, intraindividual crossover comparison of gadobenate dimeglumine and gadopentetate dimeglumine for Breast MR imaging (DETECT Trial). Radiology 2011; 258: 396 - 408

21 Epstein JI. An update of the Gleason grading system. J Urol 2010; 183: $433-440$

22 Wang HZ, Riederer SJ, Lee JN. Optimizing the precision in T1 relaxation estimation using limited flip angles. Magn Reson Med 1987; 5: 399416

23 Walker-Samuel S, Leach MO, Collins DJ. Reference tissue quantification of DCE-MRI data without a contrast agent calibration. Phys Med Biol 2007; 52: 589-601

24 Tofts PS, Kermode AG. Measurement of the blood-brain barrier permeability and leakage space using dynamic MR imaging. 1. Fundamental concepts. Magn Reson Med 1991; 17: 357-367

25 Kuhl CK, Mielcareck P, Klaschik S et al. Dynamic breast MR imaging: are signal intensity time course data useful for differential diagnosis of enhancing lesions? Radiology 1999; 211: 101 - 110
26 Link TM, Stahl R, Woertler K. Cartilage imaging: motivation, techniques, current and future significance. Eur Radiol 2007; 17: 1135-1146

27 Kim ES, Chang JH, Choi HS et al. Diagnostic yield of double-dose gadobutrol in the detection of brain metastasis: intraindividual comparison with double-dose gadopentetate dimeglumine. Am J Neuroradiol 2010; 31: $1055-1058$

28 Anzalone N, Gerevini S, Scotti $R$ et al. Detection of cerebral metastases on magnetic resonance imaging: intraindividual comparison of gadobutrol with gadopentetate dimeglumine. Acta Radiol 2009; 50: 933 940

29 Schlemmer HP. Multiparametrische MRT der Prostata: Methode zur Früherkennung des Prostatakarzinoms? Fortschr Röntgenstr 2010; 182: $1067-1075$

30 Franiel T. Multiparametrische Magnetresonanztomografie der Prostata - Technik und klinische Anwendungen. Fortschr Röntgenstr 2011; 183: $607-617$

31 Beyersdorff D, Ludemann L, Dietz E et al. Dynamische kontrastmittelunterstützte MRT der Prostata: Vergleich von zwei Auswerteverfahren. Fortschr Röntgenstr 2011; 183: 456-461

32 Barentsz JO, Richenberg J, Clements R et al. ESUR prostate MR guidelines 2012. Eur Radiol 2012; 22: $746-757$

33 Rothke M, Blondin D, Schlemmer HP et al. PI-RADS-Klassifikation: Strukturiertes Befundungsschema für die MRT der Prostata. Fortschr Röntgenstr 2013; 185: 253-261

34 Vassiou K, Kanavou T, Vlychou $M$ et al. Characterization of breast lesions with CE-MR multimodal morphological and kinetic analysis: comparison with conventional mammography and high-resolution ultrasound. Eur J Radiol 2009; 70: 69-76 